\section{$\underset{\substack{\text { hommes } \\ \text { \& migrations }}}{ }$}

\section{Hommes \& migrations}

Revue française de référence sur les dynamiques

migratoires

$1324 \mid 2019$

Religion et discrimination

\title{
La rentrée du prix littéraire de la Porte Dorée (2018 -2019)
}

\section{Nihad Jnaid}

\section{(2) OpenEdition \\ Journals}

Édition électronique

URL : https://journals.openedition.org/hommesmigrations/8865

DOI : 10.4000/hommesmigrations.8865

ISSN : 2262-3353

Éditeur

Musée national de l'histoire de l'immigration

\section{Édition imprimée}

Date de publication : 1 janvier 2019

Pagination : 167-169

ISBN : 978-2-919040-44-5

ISSN : $1142-852 X$

\section{Référence électronique}

Nihad Jnaid, «La rentrée du prix littéraire de la Porte Dorée (2018 - 2019) », Hommes \& migrations [En ligne], 1324 | 2019, mis en ligne le 01 janvier 2019, consulté le 06 janvier 2022. URL : http:// journals.openedition.org/hommesmigrations/8865; DOI : https://doi.org/10.4000/ hommesmigrations.8865 


\section{La rentrée du prix littéraire de la Porte Dorée (2018-2019)}

Nihad Jnaid,

coordinatrice du prix.

Le prix littéraire de la Porte Dorée célèbre en avril 2019 ses dix ans d'existence: dix éditions d'un prix qui n'a jamais démérité par le choix des lauréats et la qualité des dizaines de romans sélectionnés chaque année.

$\mathrm{n}$ attendant de dévoiler le nom de l'heureux lauréat 2019 lors de la soirée de remise du prix prévue le jeudi 18 avril prochain, le comité de lecture du Palais ne chôme pas. Lectures et échanges ponctuent ses réunions de travail à la recherche de nouvelles pépites éditoriales, comme ce très beau roman, Le silence du chœur, de Mohammed Mbougar Sarr paru chez Présence africaine, choisi en 2018 à l'unanimité par le jury présidé par Jean-Christophe Rufin.

Au fil des mois, la liste des parutions depuis septembre 2018 s'est enrichie, avec son lot de premiers romans, de romans d'auteurs confirmés, annonçant un travail collectif de présélection particulièrement dense. La rentrée littéraire foisonnante a confirmé cette tendance, déjà analysée ces dernières années, sur la représentation des thématiques migratoires et des récits d'exil dans la littérature d'expression française. Tendance qui nous étonne toujours autant par la diversité des approches, des histoires et des styles mobilisés. Sur les 27 titres de départ, 20 continuent à être en lecture. Quelque cinq nouveaux titres viendront enrichir cette liste en janvier 2019.

Outre le comité de lecture du Palais, les élèves de trois lycées des trois académies de Créteil, Paris et Versailles participent au Prix. Au lycée Eugène Hénaff de Bagnolet, c'est un groupe de seconde qui s'est mis au travail début décembre avec cinq titres, tandis que les élèves de première $L$ du lycée Jacques Decour, à Paris, vont mettre à profit les vacances de fin d'année pour découvrir les cinq autres titres choisis par leur professeure. La troisième classe, une seconde du lycée Jules Verne de Cergy, se joint à cette dynamique littéraire dès la rentrée des classes en janvier 2019. 


\section{Multiplicité des récits}

De nouveau, depuis septembre 2018, les formes romanesques empruntées par les romans parus couvrent un large spectre de possibilités. Aux récits autobiographiques, très nombreux dans cette présélection, se mêlent les récits fictionnels qui nous plongent dans une grande richesse d'espaces et de temporalités imaginaires. Lorsqu'Adrien Bosc opte pour un roman prenant la forme d'un journal de bord relatant la traversée du Capitaine-Paul-Lemerle, répondant au cadre spatial de l'histoire racontée qui se déroule sur un bateau, Émilie de Turckheim présente un journal qui relate le séjour d'un jeune homme réfugié afghan au sein de sa famille française une année durant. Max Lobe, écrivain camerounais résident en Suisse, choisit quant à lui les chiffres romains pour numéroter ses chapitres racontant le rêve avorté de deux jeunes Africains quittant leur pays pour l'Europe, tout comme David Diop qui nous plonge dans l'horreur des combats au front lors de la Première Guerre mondiale vécue dans la souffrance et la violence extrême d'un tirailleur sénégalais. Estelle-Sarah Bulle préfère pour sa part les chapitres à

Sur les 27 titres de départ, 20 continuent à être en lecture. Quelque cinq nouveaux titres viendront enrichir cette liste en janvier 2019. titre - « Petit-Frère 》, répété plusieurs fois, «Antoine: prospérité» ou « Antoine: second départ 》 ou bien « La nièce 》 - pour raconter l'histoire de la famille Ezechiel, et à travers elle, celle de la Guadeloupe et de toute une génération d'Antillais.

Et si la structure adoptée par Lenka Hornakova-Civade pour son roman, Une verrière sous le ciel, est surprenante tant elle épouse parfaitement les thèmes de l'apprentissage et de la liberté pour raconter comme « un tableau » les vies de la jeune pragoise, Ana, celle inventée par Gauz n'en est pas moins originale pour aborder une vaste et ambitieuse épopée coloniale en Afrique à la fin du XIX siècle croisée avec le retour d'un enfant auprès de sa grand-mère africaine. Chez Hornakova-Civade, on retrouve une division classique en "Livre» au nombre de trois, et chacun de ces livres est divisé à son tour en séquences numérotées. Le roman se termine par un chapitre intitulé « Lignes de suite ». Gauz, lui, préfère nommer ses chapitres avec des titres surprenants et provocateurs "Chapitre rouge, Chapitre urbain 》, par des «légendes 》 pas moins surprenantes « Légende de la première débarquée ou Légende de l'interprète zéro ». Une démarche inattendue qui accompagne ces allers-retours entre deux continents, l'Europe et l'Afrique, à un siècle d'écart.

\section{D'un continent à l'autre}

Cette diversité de formats de présentation reflète la forte amplitude géographique des romans pour traiter des thématiques du prix de l'exil, des migrations et des problématiques attenantes. Ainsi, dans les romans de cette présélection, personnages et destins se croisent en passant d'un continent à l'autre. L'Afrique est un point de départ très présent pour cette rentrée littéraire, pour Jean et Simon qui tentent de faire le «boza » dans Loin de Douala de Max Lobe, pour Alfa Ndiaye et Mademba Diop dans Frère d'âme..., elle est aussi le point d'arrivée 
de Dabilly dans Camarade Papa de Gauz et le fief de la famille de Penda dans Je suis quelqu'un d'Aminata Aidara. Le Proche et le Moyen-Orient ne sont pas en reste avec Les exilés meurent aussi d'amour ou Des ailes au loin. De l'autre côté de l'océan Atlantique, les Antilles offrent un cadre de prédilection à Capitaine d'Adrien Bosc et à Là où les chiens aboient par la queue de Estelle-Sarah Bulle. Néanmoins, deux titres échappent à ce cadre géographique et laissent libre cours à l'imagination du lecteur : Salina : les trois exils et L'Archipel du chien.

Trois titres au moins abordent spécifiquement des thèmes ancrés dans la société française d'aujourd'hui. Dans Jeune fille modèle, l'auteure-blogueusecinéaste Grace Ly rend hommage à sa mère et présente le point de vue de cette seconde génération d'immigrés asiatiques qui tente de déconstruire les stéréotypes « anti-asiatique ». C'est le seul roman de cette rentrée qui aborde de front les questions d'appartenance et la façon d'accepter sa différence physique lorsqu'on est issu d'une minorité, les Chinois du Cambodge - «Chi Chi est une jeune fille comme les autres » -, tout en assumant une identité culturelle différente, celle que défend Grace Ly à travers son engagement militant.

Tu n'habiteras jamais Paris d'Omar Benlaala nous amène du $13^{\mathrm{e}}$ au $20^{\mathrm{e}}$ arrondissement de Paris, et tout particulièrement dans le quartier de Ménilmontant. L'auteur fait parler son père un an durant pour raconter son histoire, familiale certes, mais également l'histoire d'un Paris disparu. Cet hommage au père est empreint de tendresse. Il dit sa gratitude et sa fierté à l'égard de ce père qui a osé faire des choix courageux, de l'apprentissage de la langue aux multiples efforts d'intégration, à la persévérance et la force des convictions. Un récit à double voix qui fait le parallèle entre Bouzid Benlaala, maçon kabyle, et Martin Nadaud, maçon de la Creuse.

Le troisième titre est celui d'Émilie de Turckheim, Le Prince à la petite tasse, un témoignage d'une expérience personnelle et familiale qu'a vécue l'auteure avec ses deux enfants et leur père. Son journal relate le séjour d'un jeune afghan, Reza, accueilli durant un an dans l'appartement parisien de la famille. C'est un récit sur l'hospitalité et l'accueil quand il devient une réalité quotidienne. Un récit sur les relations humaines, la curiosité qui nous anime et nous pousse vers l'autre: "J'ai mille questions à lui poser [...] Je n'en pose aucune. 》 Une note d'optimisme et de générosité se dégage de cette formidable aventure où la «fraternité » n'est pas un vain mot.

Ces romans offrent les diverses facettes de notre monde. Un monde fait de rencontres, de déplacements et de métissage. À travers ces fictions, tour à tour, la littérature nous plonge dans les réalités contemporaines de plusieurs continents lointains et met à jour un pan de l'histoire, interroge nos rapports à l'Autre et nous livre une lecture romanesque des grandes questions d'actualité. 


\section{LA PRÉSÉLECTION DU PRIX LITTÉRAIRE DE LA PORTE DORÉE 2019}

- Aminata Aidara, Je suis quelqu'un, Gallimard

- Omar Benlaala, Tu n'habiteras jamais Paris, Flammarion

- Adrien Bosc, Capitaine, Stock

- Estelle-Sarah Bulle, Là où les chiens aboient par la queue, Liana Levi

- Philippe Claudel, L'Archipel du chien, Stock

- Benoît Cohen, Mohammad, ma mère et moi, Flammarion

- David Diop, Frère d'âme, Seuil

- Laurent Gaudé, Salina: les trois exils, Actes Sud

- Gauz, Camarade Papa, Le nouvel Attila - Lenka Harnakova-Civade, Une verrière sous le ciel, Alma éditeur
- Jadd Hilal, Des ailes au Loin, Elyzad

- Alain Jomy, Olga et les siens, Alma éditeur

- Max Lobe, Loin de Douala, Zoé

- Grace Ly, Jeune fille modèle, Fayard

- Sarah Marty, Soixante jours, Denoël

- Scholastique Mukasonga, Un si beau diplôme, Gallimard

- Tobie Nathan, L'Évangile selon Youri,

Stock

- Patrice Nganang, Empreintes de crabe, JC Lattès

- Abnousse Shalmani, Les exilés meurent aussi d'amour, Grasset - Émilie de Turckheim, Le Prince à la petite tasse, Calmann-Lévy 